\title{
Atrapadas en una espiral de precariedad y exclusión: trayectorias de mujeres inmigrantes en programas residenciales para mujeres con menores a cargo ${ }^{1}$
}

\author{
Nerea Layna Allue
}

Facultad de Psicología y Educación, Universidad de Deusto

\section{Itziar Gandarias Goikoetxea}

Facultad de Psicología y Educación, Universidad de Deusto

\section{Miguel Ángel Navarro Lashayas}

Facultad de Psicología y Educación, Universidad de Deusto

miguel.navarro@deusto.es

\begin{abstract}
Artikulu honen helburua emakume etorkinak Euskadira iristetik bete dituzten ibilbideak aztertzea da. Horretarako, adingabeak ardurapean dituzten emakumeentzako egoitza-programa batetik pasatzeak izandako eraginak, bizipenak eta mugak azaltzen dira, baita egungo egoera ekonomikoak, laboralak eta egoitza-egoerak ere. Errealitate hori ezagutzeko, hamabost elkarrizketa sakon egin zaizkie azken hamabost urtetan baliabidearen erabiltzaile izandako emakume etorkinei.

Azterketaren ondorio nagusiak honako hauek dira, besteak beste: ezkutuko etxegabetasunaren presentzia nabarmena, eta kalean geratzeari aurre emateko emakumeen arteko harreman-estrategien presentzia; lana sektore feminizatu oso prekario eta ezegonkorretara mugatzea, prekarietatearen eta bazterkeriaren etengabeko espiraletik irtetea ahalbidetzen ez dietenak; baita ere irismen luzeko programen garrantzia, lanaz haraindi, laneratzearen ikuspegi holistiko eta intersekzionala eta lagunegite psikologikoa eta indarkeria anizkoitzen konponketa bilatzen dutenak.
\end{abstract}

\section{GAKO-HITZAK:}

Emakume etorkinak, ezkutuko etxegabetasuna, gizarte-bazterketa, lan-prekarietatea.
Este artículo tiene como objetivo analizar las diversas trayectorias de mujeres inmigrantes desde su llegada al País Vasco. Para ello, se observan los efectos, vivencias y limitaciones de su paso por un programa residencial para mujeres con menores a cargo, así como su situación económico-laboral y residencial actual. Para conocer esta realidad, se han realizado quince entrevistas en profundidad a mujeres inmigrantes que fueron usuarias del recurso durante los últimos quince años. Las principales conclusiones derivadas del análisis son, entre otras, la fuerte presencia del sinhogarismo encubierto y de estrategias relacionales entre las mujeres para no acabar en la calle; el enclaustramiento laboral en sectores feminizados muy precarios e inestables que no les permiten salir de una continua espiral de precariedad y exclusión, así como la importancia de programas de largo alcance con una mirada holística e interseccional de la inclusión social más allá del ámbito laboral, que contemplen el acompañamiento psicológico y la reparación de las múltiples violencias.

\section{Palabras Clave:}

Mujeres inmigrantes, sinhogarismo oculto, exclusión social, precariedad laboral. 


\section{Introducción}

Los fenómenos de la exclusión social y el sinhogarismo han sido ampliamente investigados en los últimos años (Brugué, Gomà y Subirats, 2014; Fundación Atenea, 2018; Foessa, 2019). Si bien se ha concluido que se trata de una cuestión que es necesario analizar a partir de una mirada multifactorial y multicausal, es evidente que la situación específica de las mujeres ha quedado tradicionalmente relegada a un segundo plano, entendiendo que los resultados de los diferentes abordajes que se han llevado a cabo son generalizables tanto para hombres como para mujeres.

Sin embargo, es necesario señalar que la gran mayoría de estudios se han realizado a través de experiencias relatadas por hombres, siendo estos los usuarios mayoritarios de los recursos destinados para las personas sin hogar (Matulič, De VicenteZueras, Boixadós-Porquet y Caïs-Fontanella, 2019; Fernández- Rasines y Gámez- Ramos, 2012). De esta manera, a partir de una supuesta neutralidad en los resultados obtenidos, sistemáticamente se han invisibilizado problemáticas y necesidades específicas asociadas a las mujeres. De hecho, el sinhogarismo sigue siendo un fenómeno impregnado de un imaginario predominantemente masculino asociado a enfermedades mentales, drogodependencias y hombres mayores (Arrels, 2017), a pesar de que estudios recientes muestran una gran heterogeneidad y un incremento en el número de jóvenes y de mujeres en situación de sin hogar (Ministerio de Sanidad, Consumo y Bienestar Social, 2015-2020).

En los últimos años, la cuestión de las mujeres sin hogar ha supuesto un fenómeno central en estudios europeos sobre sinhogarismo, no solo para desagregar datos cuantitativos diferenciados de hombres y mujeres, sino para desarrollar y fomentar una perspectiva de género crítica que ayude a dar explicación tanto al menor número de mujeres en situación de sin hogar como a los diferentes motivos estructurales, sociales y relacionales que están detrás de esta invisibilización. Estos estudios revelan cómo al acotar el fenómeno del sinhogarismo únicamente a las personas que se encuentran en calle o que duermen en recursos y albergues de noche, la situación específica de las mujeres queda totalmente invisibilizada (Pleace, 2016; Bizitegi, 2018).

En el caso del Estado español, los últimos informes de Foessa $(2014,2019)$, ponen de manifiesto el incremento de mujeres inmigrantes en situación de sinhogarismo y una problemática específica y compleja fruto del cruce de las divisiones sociales de género, clase, origen nacional, raza y condición jurídica como extranjeras, que determina y condiciona una respuesta eficaz e integral por parte de los programas de intervención social. De esa manera, se torna necesario conocer en mayor profundidad las múltiples situaciones de vulnerabilidad que enfrentan las mujeres inmigrantes. Por ello, este artículo se plantea como objetivo analizar las diversas trayectorias de quince mujeres inmigrantes desde su llegada al País Vasco. Para ello, se exploran los efectos, vivencias y limitaciones de su paso por un programa específico residencial para mujeres con menores a cargo, así como su situación económica-laboral y residencial actual.

\section{Sinhogarismo oculto}

\subsection{Cuando techo y hogar no son lo mismo}

Si atendemos la dimensión de la vivienda como el espacio central de desarrollo y autonomía de las personas y como factor principal de la exclusión social por delante del desempleo (Foessa, 2019), es evidente que las mujeres tienen una mayor dificultad para mantener una vivienda digna y estable debido a un menor poder adquisitivo respecto a los hombres. Según el Instituto Nacional de Estadística (2012) la presencia femenina aumenta en las categorías vinculadas a la exclusión residencial de la tipología ETHOS (vivienda insegura y vivienda inadecuada), siendo su representación del $48 \%$ y manteniendo una presencia mucho menor en las categorías de falta de vivienda (sin techo y sin vivienda).

La redefinición de lo que entendemos por hogar y por techo ha sido uno de los aportes más importantes en torno al fenómeno del sinhogarismo desde una perspectiva de género (Fernández y Gámez, 2013; Carrasco, 2014 y Matulič et al., 2019). El techo es un lugar donde las personas pueden estar a cubierto y alojarse. Sin embargo, esto no implica que las personas se sientan seguras, ni que existan sentimientos de pertenencia o integración. Un hogar, sin embargo, es un lugar donde las personas, además de sentirse seguras, tienen control sobre el espacio, autonomía y privacidad, y donde puede desarrollar su bienestar personal y social. Teniendo en cuenta esto, es posible que la persona sin hogar tenga una vivienda disponible pero que esta no contenga condiciones seguras y dignas. Acín (2016) lo resume de esta manera: "todos/as los/as sin techo son sin hogar, pero no todos/as los/as sin hogar son sin techo".

Las desigualdades de género tienen un impacto directo en las limitaciones que encuentran las mujeres para sostener hogares independientes (Edgar y Doherty, 2001; Sales y Guijarro, 2017). Estas desigualdades generan itinerarios y estrategias diferenciadas que las mujeres realizan respecto a los hombres, conformando un sinhogarismo oculto (Enders-Dragässer, 2010) que transcurre en el ámbito privado, de ahí que sea un fenómeno opaco difícil de percibir y visibilizar.

Las mujeres, por sus atribuciones de género, mantienen vínculos que les permiten esquivar la pernocta a la intemperie. Como señala Martins 
(2010), las mujeres consideran otras formas de protección distintas a las de los hombres, vinculadas a su relación con familiares y personas conocidas en mayor o menor grado. El uso de esta estrategia las previene de acabar en calle o del uso de servicios de alojamiento para la población sin hogar en general (Fernández-Rasines y Gámez-Ramos, 2013).

Sales y Guijarro (2017) sostienen que, a pesar de que muchas mujeres en situación de alta vulnerabilidad se encuentran con escasa red de apoyo, el mantenimiento de ciertas relaciones y la mayor facilidad para pedir ayuda (propia de la socialización femenina) facilitan que las mujeres puedan quedarse en viviendas o habitaciones de su red informal de apoyo. No obstante, en ocasiones, donde los vínculos sociales y familiares son frágiles, se han perdido, o se encuentran lejos, como es el caso de la inmigración, las mujeres desarrollan otras estrategias para impedir quedarse en la calle, como intercambiar sexo o cuidados a cambio de un techo prestado. Estas estrategias muy invisibles y atravesadas por las desigualdades de género (Baptista y Marlier, 2019) resultan una especificidad del sinhogarismo femenino que necesita ser estudiada y abordada en profundidad de cara a una compresión más compleja de la menor presencia de mujeres en calle.

\subsection{Mujeres inmigrantes, monomarentalidad y sinhogarismo}

Los últimos estudios de Foessa $(2014,2019)$ muestran que uno de los grupos más vulnerables en situación de sinhogarismo son las mujeres inmigrantes, ya que a las dificultades de acceso a la vivienda y el empleo se enfrentan a los obstáculos que supone estar en situación de irregularidad administrativa y las escasas o frágiles redes de apoyo con las que cuentan (Matulič et al., 2020).

Cuando además existen menores a cargo, la situación de exclusión puede agravarse. Investigaciones realizadas en los últimos años señalan que los hogares monoparentales sustentados por mujeres se encuentran en desventaja respecto al resto. Por ejemplo, en estudios europeos realizados por Pleace (2016) un $25 \%$ de familias encabezadas por mujeres vive en situación de pobreza, y cuatro de cada diez se encuentran en riesgo. En el caso del Estado español, la dimensión económica de la exclusión social afecta en mayor medida a hogares liderados por mujeres y los datos más altos en la mayoría de indicadores provienen de hogares monoparentales; donde más que de familias monoparentales habría que hablar de familias "monomarentales", ya que el 86,2\% de los casos el progenitor responsable es la madre (Foessa, 2019). El análisis efectuado demuestra asimismo la existencia de una relación estrecha entre monoparentalidad y procesos de exclusión. Esto se aprecia, ante todo, en el hecho de que el Índice Sintético de Exclusión Social (ISES) ${ }^{2}$ de esta tipología

${ }^{2}$ El índice sintético de exclusión social (ISES) analiza la exclusión de hogares es más que el doble que el del resto de hogares $(2,7$, frente al 1,3 del conjunto de hogares cuya sustentadora principal es una mujer y al 1,2 de aquellos cuyo sustentador principal es un hombre).

Desde una mirada cualitativa, Damonti (2014) y Morante et al. (2010) ahondaron en las barreras a las que se enfrentan las mujeres sin hogar. En primer lugar, identificaron que la mayoría de limitaciones están asociadas al mercado laboral (un menor acceso al mismo y dificultades para el sostenimiento económico), a los roles de género que vinculan los cuidados de familiares y personas dependientes a las mujeres y, por último, a una mayor vulnerabilidad ante situaciones de violencia. De la misma manera, los informes Foessa $(2014,2019)$ señalan que la pobreza de las mujeres tiene una vinculación directa con la posición de subordinación en el mercado de trabajo, ya que ocupan mayoritariamente puestos laborales caracterizados por la precariedad, el desprestigio social y los bajos salarios.

En el caso específico de las mujeres inmigrantes, esta posición de subordinación es fruto de la articulación de las divisiones sociales de género, clase, origen nacional, raza, etnicidad y religión, así como también de su condición jurídica como extranjeras, que condiciona y determina el acceso a derechos y oportunidades sociales y laborales (Anthias, 1998). Laura Oslo y Sonia Parella (2013) encontraron que las mujeres inmigrantes tienen un mayor enclaustramiento en trabajos no cualificados y un mayor estancamiento laboral si se comparan con sus pares hombres.

El servicio doméstico y de cuidados es el primer nicho laboral que mayoritariamente encuentran las mujeres a su llegada y se mantiene pasado los años, lo cual las hace vivir en situaciones de alta inestabilidad y precariedad laboral sin poder configurar un hogar seguro y estable para ellas y sus familias. De hecho, un estudio cualitativo realizado por Parella y Samper (2008), señala los obstáculos adicionales a los que se enfrentan las trabajadoras inmigrantes monomarentales a la hora de conciliar, ya que debido a los segmentos laborales que ocupan, cuentan con escasos recursos económicos para acceder a servicios privados de cuidados para sus hijos e hijas, a lo que se suman las escasas redes familiares próximas. Entre las estrategias que las mujeres ponen en marcha encontraron que muchas de ellas optan por trabajos intermitentes o por no seguir empleadas, ya que el bajo salario que reciben es mayor que el coste de externalizar el cuidado de sus criaturas.

social a partir de 35 indicadores. Es un índice basado en la identi ficación de situaciones fácticas, constatables empíricamente y de suficiente gravedad como para poner en cuestión la plena participación social de las personas afectadas. Es la acumulación de diversas situaciones de dificultad lo que sitúa a determinados grupos de la sociedad en posiciones de exclusión del espacio social (Foessa, 2019). 


\section{El programa Mujer en situación de exclusión social con menores acompañados, de Lagun Artean ${ }^{3}$}

El programa Mujer en situación de exclusión social con menores acompañados de la organización Lagun Artean de Bizkaia inició su recorrido en el año 2004, con un total de cuatro recursos residenciales destinados a estas familias. Son viviendas de media intensidad educativa destinadas a mujeres mayores de edad solas o con menores a cargo. En total es una red de cuatro viviendas con un total de 19 plazas, 11 para mujeres y 8 para menores, ubicadas en el barrio de Deusto. El programa se caracteriza por una ocupación media del $95 \%$ de sus plazas (Asociación Lagun Artean, 2018).

Durante los quince años de recorrido de este programa, se ha atendido a un total de 118 mujeres y 114 menores. La Tabla 1 presenta las características descriptivas de las mujeres atendidas.

Tabla 1. Datos descriptivos de las mujeres y las/os menores atendidas en Lagun Artean

\begin{tabular}{|c|c|c|}
\hline & $N=118$ & Porcentaje \\
\hline \multicolumn{3}{|l|}{ Origen } \\
\hline Marruecos & 28 & $23,7 \%$ \\
\hline África Subsahariana & 60 & $50,7 \%$ \\
\hline Centroamérica & 20 & $17,3 \%$ \\
\hline España & 9 & $7,6 \%$ \\
\hline Asia & 1 & $0,7 \%$ \\
\hline \multicolumn{3}{|l|}{ Edad $(\overline{\mathrm{X}}=38,9)$} \\
\hline $19-29$ & 17 & $14,4 \%$ \\
\hline $30-39$ & 50 & $42,4 \%$ \\
\hline 40-49 & 39 & $33 \%$ \\
\hline $50-59$ & 9 & $7,6 \%$ \\
\hline $60-69$ & 2 & $1,7 \%$ \\
\hline 70-79 & 1 & $0,85 \%$ \\
\hline \multicolumn{3}{|c|}{ Tiempo de estancia en meses $(\bar{X}=\mathbf{8}, 5)$} \\
\hline Entre o y 6 meses & 52 & $50 \%$ \\
\hline Entre 6 meses y 1 año & 25 & $24 \%$ \\
\hline Entre 1 año y 1 y medio & 17 & $16.3 \%$ \\
\hline Entre 1 y medio y 2 años & 6 & $5.8 \%$ \\
\hline Entre 2 años y 2 medio & 2 & $1.9 \%$ \\
\hline Entre 2 y medio y 3 años & 1 & $0.9 \%$ \\
\hline Entre 3 años y 3 y medio & 1 & $0.9 \%$ \\
\hline Número de hijas/os* & $N=115$ & Porcentaje \\
\hline 0 & 28 & $24,6 \%$ \\
\hline 1 & 44 & $38,6 \%$ \\
\hline 2 & 22 & $19,3 \%$ \\
\hline 3 & 11 & $9,6 \%$ \\
\hline 4 & 4 & $3,5 \%$ \\
\hline 5 & 4 & $3,5 \%$ \\
\hline 6 & 1 & $0,9 \%$ \\
\hline 7 & 1 & $0,9 \%$ \\
\hline
\end{tabular}

* El número de hijas/os hace referencia a los que están tanto en origen como en destino.

Fuente: Datos de Intervención Lagun Artean.

${ }^{3}$ Lagun Artean es una asociación vinculada a Cáritas Diocesana de Bilbao que acompaña los procesos de incorporación social de las personas sin hogar y otras personas en situación de grave exclusión social y de riesgo de gran exclusión. Para más información: http:// www.lagun-artean.org/.
En cuanto al origen de las mujeres atendidas, se observa que la gran mayoría de ellas procede del continente africano, concretamente de Marruecos y África subsahariana. En segundo lugar, se encuentran las mujeres procedentes de Centroamérica, seguidas de las españolas. Las mujeres asiáticas conforman una minoría dentro de las 118 mujeres atendidas, contando únicamente con un caso. Atendiendo a los rangos de edad, cabe destacar que la gran mayoría de ellas se encuentran entre los 30 y 39 años de edad, seguidas por las que tienen entre 40 y 49 años.

Teniendo en cuenta el tiempo de estancia media en meses, es significativo afirmar que el $50 \%$ de las mujeres atendidas estuvieron entre o y 6 meses, seguidas de un $\mathbf{2 4} \%$ de ellas que fueron atendidas durante un total de entre seis meses a un año. Los cortos plazos de intervención se deben a la alta demanda de mujeres que el programa residencial de Lagun Artean presenta de manera constante.

En relación con el número de hijas/os, la mayoría de las mujeres atendidas tiene un menor a su cargo, un $40 \%$, casi un $20 \%$ tiene dos y cerca del $10 \%$ tres hijos/as, mientras que un $24,6 \%$ del total no tenía ninguno. En menor medida, casi un $8 \%$ de las mujeres tiene cuatro o más hijos. Es importante destacar que el número de hijas/os a cargo hace referencia a los/as que estaban tanto en el país de origen como en destino.

\section{Metodología y participantes}

Esta investigación se ha desarrollado en diferentes fases. En un primer momento, se llevó a cabo una sistematización cuantitativa de datos relevantes de las 118 mujeres atendidas en el programa Mujer de Lagun Artean, tal y como se puede ver en la Tabla 1. En base a esta sistematización y teniendo en cuenta el objetivo de la investigación -analizar los itinerarios de las mujeres inmigrantes antes, durante, y después de su estancia en el programase diseñaron los perfiles y se construyó un guion para la entrevista. Como el perfil de las 118 mujeres atendidas en el programa ha sido muy heterogéneo a lo largo de los quince años de intervención, se ha intentado respetar la diversidad de las quince mujeres entrevistadas. Para ello, se han tenido en cuenta aspectos como la procedencia, la edad o la situación sociolaboral en el momento de la realización de la entrevista.

La técnica cualitativa empleada ha sido la entrevista semiestructurada en profundidad (Î̃niguez, 1999). La duración media de las entrevistas fue de una hora y todas ellas fueron grabadas previo consentimiento por parte de las participantes. Mediante las entrevistas se ha procedido a explorar las trayectorias diversas de estas mujeres en base a un guion previo, pero a la vez dando pie a la improvisación y desde una posición no directiva, en la que las propias personas participantes se 
Tabla 2. Perfiles de las mujeres participantes en la investigación

\begin{tabular}{|c|c|c|c|c|}
\hline Mujer & Origen & Edad & Año en que utilizaron el recurso & Hijas/os \\
\hline 1 & Nicaragua & 42 & 2011 & Sí \\
\hline 2 & Mali & 49 & 2007 & No \\
\hline 3 & Nigeria & 35 & 2011 & Sí \\
\hline 4 & Bolivia & 39 & 2012 & Sí \\
\hline 5 & Mali & 32 & 2012 & Sí \\
\hline 6 & Senegal & 42 & 2013 & Sí \\
\hline 7 & Argelia & 43 & 2014 & No \\
\hline 8 & Sáhara & 33 & 2016 & Sí \\
\hline 9 & Marruecos & 39 & 2012 & No \\
\hline 10 & Camerún & 25 & 2013 & No \\
\hline 11 & Guinea Bissau & 44 & 2017 & Sí \\
\hline 12 & Nicaragua & 38 & 2007 & Sí \\
\hline 13 & Sahara & 37 & 2011 & Sí \\
\hline 14 & Guinea Ecuatorial & 40 & 2014 & Sí \\
\hline 15 & Senegal & 38 & 2009 & Sí \\
\hline
\end{tabular}

Fuente: Elaboración propia.

pudieran sentir libres para expresarse de manera espontánea sobre algún tema que les surgiera (Taylor y Bogdan, 1992). Como señala Olabuenaga (2012) la metodología cualitativa permite un mejor acceso a narrativas personales, posibilitando después una mayor profundidad de análisis con el material recolectado.

En la Tabla 2 se presenta las características principales del perfil de las quince mujeres entrevistadas.

\section{Análisis de los resultados}

\subsection{Atrapadas en un círculo continuo de precariedad y exclusión}

En el Gráfico 1 se recogen los aspectos más importantes recabados del análisis de las entrevistas. El análisis se ha estructurado en cuatro áreas que en los siguientes apartados analizaremos en profundidad: (1) el perfil y las características comunes de las mujeres que llegan al programa; (2) aportes y limitaciones

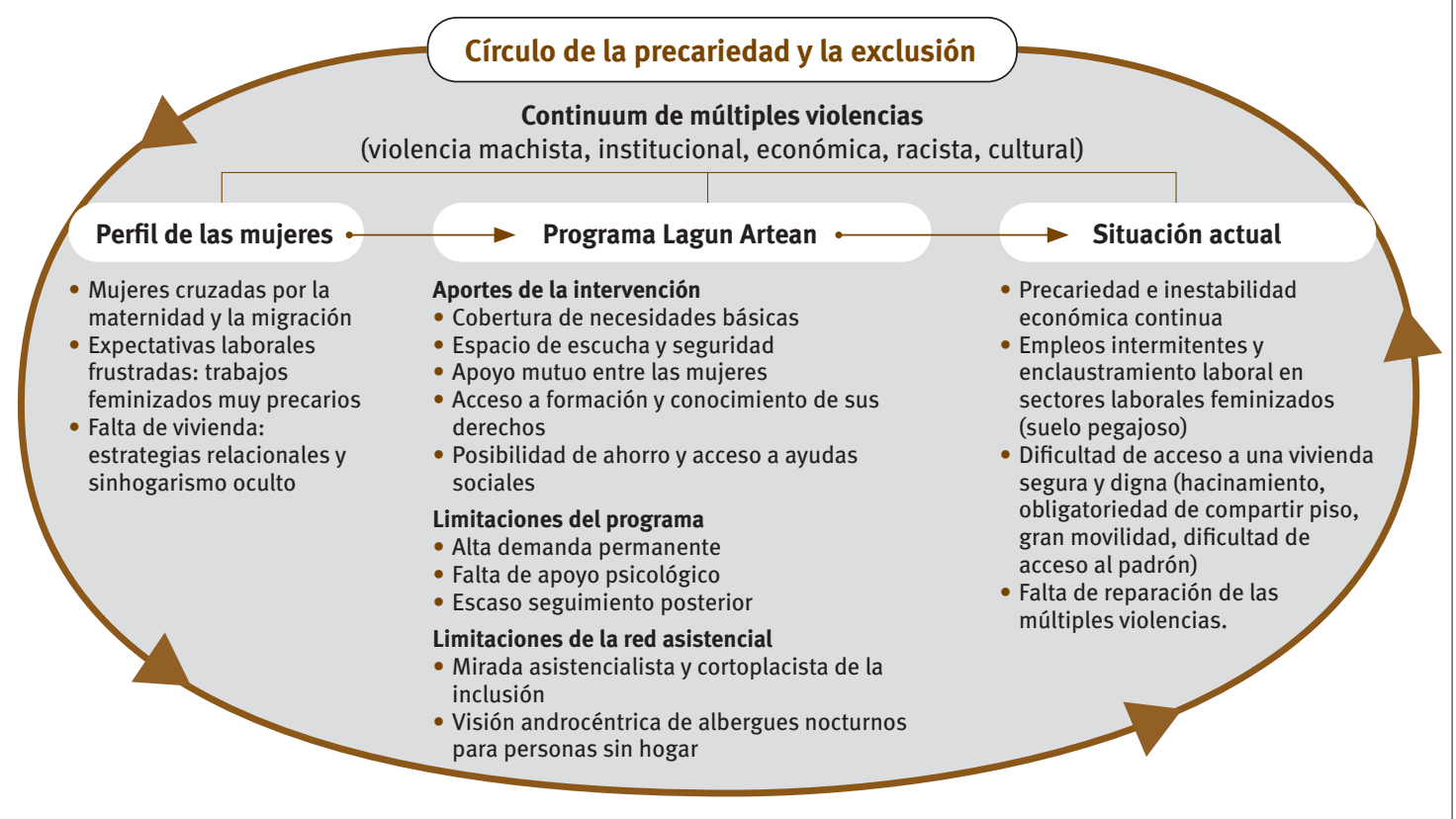

Fuente: Elaboración propia. 
del programa y de la red asistencial a partir de la experiencia de las mujeres; y (3) la situación actual de las mujeres incidiendo en dos aspectos clave para la inclusión social: el ámbito económico-laboral y la vivienda. Por último, y en menor medida, se analiza (4) el continuum de las múltiples violencias que atraviesan las trayectorias de las mujeres.

\subsection{Perfil y características comunes de las mujeres que llegan al programa}

Presentamos a continuación el perfil de las mujeres que llegan al Programa. A pesar de la heterogeneidad de las mismas hemos identificado tres aspectos comunes que recorren las trayectorias de las mujeres: (1) el cruce de la maternidad y la migración en sus recorridos, (2) expectativas laborales frustradas y (3) falta de vivienda digna y sinhogarismo oculto.

\subsubsection{Mujeres cruzadas por la maternidad y la migración}

El 90,7\% de las mujeres atendidas por el programa de Lagun Artean en los últimos quince años son mujeres migradas y un $75,5 \%$ son madres, de las cuales el $58 \%$ tiene de media uno o dos hijos/as. En ocasiones son hijos e hijas que han dejado en sus países, otras veces los han traído con ellas y también hay mujeres que dejaron hijos en sus países y luego aquí han sido madres de nuevo. En el caso de las quince mujeres entrevistadas, once de ellas tienen hijas/os a cargo, mientras que las cuatro restantes, no.

Ser madre cruza y condiciona íntegramente la experiencia de las mujeres migradas a la hora de encontrar trabajo, ya que se encuentran más limitadas a la hora de acceder a jornadas largas de trabajo o en régimen de interna, además de que el trabajo no solo termina con la jornada laboral, sino que luego está todo el trabajo reproductivo de cuidados cuando vuelven a casa:

\section{Una mujer sin hijos trabaja donde sea, se mete interna, no tiene quien te llore, que te diga mamá tengo hambre, en cambio cuando una tiene hijos tienes que saber dónde trabajar, cómo, qué horario puedes trabajar, porque no puedes trabajar corrido cuando tienes hijos. Tienes que venir a cuidarlos, porque no se trata solo de salir a trabajar y venir a casa a descansar, llegas a tu casa y sigues trabajando porque están tus hijos, está tu familia que te está esperando. (Participante 12, Nicaragua)}

A esto hay que sumar el miedo a que les quiten sus criaturas. Tal y como señalan Matulič et al. (2020), cuando a las dificultades económicas y laborales se les suman otras como la posibilidad de perder la custodia de sus hijos/as, la carga emocional, el estrés y la urgencia de encontrar un alojamiento aumentan:
Cuando llegué a Lagun Artean, la primera vez que hablé con la educadora social, lo más importante para mí era hacer algo, buscar algo donde vivir, tenía miedo por si me quitaban a mi hijo. (Participante 8, Sáhara)

Para las mujeres que han dejado a sus hijos/as en sus países de origen y han realizado el proyecto migratorio de manera solitaria, la situación tampoco es fácil. La incertidumbre de no saber si van a volver a verles y los sentimientos de culpa se entremezclan y les acompañan durante todo su proceso migratorio y estancia en los países de destino:

Dejé a mis dos hijos, se quedaron allá, aquí tuve del que venía embarazada [...] dejarlos allí fue muy difícil, eso es lo más horrible que le puede pasar a una mujer [...] no sabes ni si vas a regresar, si vas a volver a verlos... (Participante 12, Nicaragua)

\subsubsection{Expectativas laborales frustradas: abocadas a trabajos feminizados}

La reorganización global del cuidado sitúa a las mujeres migrantes en una situación de marcada vulnerabilidad laboral y social y las aboca a nichos laborales específicos, que como señalan Oso y Parella (2012) se convierten para muchas de ellas en la única "puerta de entrada" al mercado de trabajo en España. Estas autoras identifican dos nichos de trabajo principales: el trabajo doméstico remunerado y el trabajo sexual.

Sin embargo, las mujeres vienen con unas expectativas diferentes, pensando que las opciones de trabajo son más diversas y sobre todo que las condiciones son mejores, lo que les va a permitir poder ahorrar rápidamente dinero y regresar lo antes posible a sus países:

Pensaba que cuando volase a Europa iba a trabajar bien y mandar dinero a mi país. Y luego volver a mi país y trabajar allí, no pensaba para nada quedarme en Europa. Pensaba que cuando llegas, te llaman y puedes elegir si quieres trabajar o no. Pero cuando llegué fue muy diferente. Pensaba que iba a trabajar en hoteles que era lo que yo quería, pero no ha sido así. Hay una diferencia demasiado grande. (Participante 15, Senegal)

Cuando llegan, las oportunidades laborales son muy limitadas y, muchas debido a su situación irregular administrativa, encuentran dificultades para acceder a trabajos en el sector de cuidados, a lo que se suma la urgencia inmediata de cubrir las necesidades básicas de sus criaturas. Es en esta situación en la que las mujeres optan por trabajar en la prostitución como única alternativa para poder mantener a sus hijos e hijas:

Hay mujeres que se prostituyen, yo estuve cuidando unas niñas de una chica que se 
prostituía para poder darle de comer a las chavalas. Te ves en una situación peor con hijos y no tienes dinero ni apoyo de nadie... las ayudas se te complican, entonces te ves en una situación súper difícil. [...] No la pasan bien porque es feo, te sientes mal cuando oyes las cosas que pasan, que viven. (Participante 12, Nicaragua)

Es así como la prostitución se puede convertir en una alternativa temporal con la que ahorrar el máximo de dinero posible, aunque como señala Laura Oso (2003, 2005), muchas acaban alargándolo durante largos periodos por las dificultades que supone salir de este sector. Por otro lado, es importante señalar que, aunque en la literatura sobre prostitución y migraciones internacionales predomina una imagen de las mujeres migrantes que ejercen prostitución como pasivas, obligadas y forzadas, es importante rescatar la agencia de las mujeres por limitada que sea para "utilizar el sexo comercial con fines instrumentales" (Agustín, 2005: 1).

En ese sentido, nos parece fundamental rescatar la dignidad de las mujeres a la hora de analizar sus situaciones y decisiones para poder luego acompañarlas desde una posición menos paternalista. Frente a discursos e imaginarios que las sitúan como mujeres pasivas, dependientes y frágiles, las mujeres se autoidentifican como mujeres trabajadoras aquí y en sus países. Subrayan la importancia que tiene para ellas su autonomía económica, y por tanto lo que les implica y supone tener que solicitar ayudas:

Nosotras, la mayoría de las personas que estamos aquí y que hemos venido aquí, casi todas son mujeres, y somos mujeres que sabemos trabajar, que hemos trabajado y que nunca hemos dependido de nadie prácticamente [...] tienes comida, tienes casa, no vives como un millonario, pero por lo menos tu comida la tienes. Y venir aquí una mujer que trabaja, mujeres que trabajaban en oficina, en diferentes cosas, que tenían su propio negocio en sus países... vienes aquí y te sientes horrible, porque pedir nunca se pide, nosotras lo ganamos, nosotras no lo pedimos. (Participante 12, Nicaragua)

\subsubsection{Falta de vivienda: estrategias relacionales y sinhogarismo encubierto}

Las mujeres entrevistadas destacan la vivienda como uno de los principales problemas a los que se enfrentan cuando llegan. No obstante, y para no acabar en la calle, las mujeres a diferencia de los hombres, utilizan estrategias relacionales (Matulič et al., 2020) como alojarse en hogares de familiares, amistades o instituciones sociales. Estas situaciones no son siempre seguras, sino que entrañan vulnerabilidades ocultas que son muy difíciles de visibilizar (Sales y Guijarro, 2017; Mayock y Sheridan, 2012).
Estas vulnerabilidades ocultas, que implican que en cualquier momento pueden quedarse en la calle, son relatadas por varias de las mujeres entrevistadas:

Yo tenía una amiga que vivía aquí y me quedaba en su casa mientras estaba en la lista para entrar al piso de Lagun Artean. Un día me dice mi amiga que me echa a la calle, no tengo donde vivir. No era muy amiga, no confiaba en mí. (Participante 3, Nigeria).

Mi madre y hermana vivían en un apartamento muy chiquitito y pues... la verdad que no se podía. Entonces me fui a casa de una amiga, contaba con el apoyo de una amiga, me fui al sofá porque no tenía más habitaciones, ella dormía con su hija y ahí estaba con mi hijo, pero puedes estar un día o dos, un tiempo, te hace el favor un tiempo. (Participante 4, Bolivia).

Como vemos, las amistades son una estrategia bastante común que utilizan las mujeres, pero son estrategias transitorias y puntuales que no les garantizan una estabilidad ni seguridad mínima. A la inestabilidad muchas veces hay que sumarle las condiciones de precariedad y hacinamiento en la que se encuentran las viviendas, teniendo que dormir en espacios minúsculos, como despensas que se alquilan al precio de habitaciones, compartiendo cama y en condiciones de insalubridad:

Una vez que llegamos a Bilbao, alquilamos una habitación por 300 euros y la chica no tenía cama, parece que era como un cuartito de despensa o algo así lo que nos alquilaba, pues ahí no entraban dos camas ni de 90 ni grande ni nada. Nos chocábamos al entrar [...]. Y ahí dormíamos, cada una en una dirección, era lo que entraba en la habitación. Era complicado porque encima hacia muchísimo frio. (Participante 12, Nicaragua)

Cuando las estrategias relaciones se agotan, las mujeres utilizan su cuerpo como recurso para no acabar sin hogar, manteniendo relaciones sexuales a cambio de una estancia:

Dormí en todos los sitios que la gente me ofrecía, tenía que hacer muchas cosas malas para poder vivir y dormir. Tenía que sobrevivir. (Participante 2, Mali)

Si van a ayudarte en algo tienen que coger más cosas, ¿sabes? De tu vida, tu cuerpo... eso. De las mujeres se aprovechan mucho. (Participante 9, Marruecos)

Todas estas situaciones de violencias y vulnerabilidades ocultas a las que se ven expuestas muestran cómo las mujeres desarrollan itinerarios diferenciados en relación con los hombres, conformando un sinhogarismo encubierto que transcurre en el espacio privado (Matulič et al., 2020) y que por tanto es más difícil de detectar. 
Aun así, las mujeres tienen claro que acabar durmiendo en calle es lo último a lo que se expondrían, ya que son conscientes de los peligros y dificultades añadidas que supone dormir a la intemperie para las mujeres:

Un hombre puede dormir en cualquier sitio, una mujer no porque está expuesta, la pueden violar, le pueden pasar mil cosas. (Participante 11, Guinea Bissau).

Fuera [en la calle] sentía miedo, ya sabes... te pueden hacer de todo. (Participante 6, Senegal).

\subsection{Aportes y limitaciones del programa Mujer en situación de exclusión social con menores acompañados}

A continuación, desarrollaremos los aportes y limitaciones que las mujeres entrevistadas identifican en torno a su experiencia en el Programa Mujer de Lagun Artean.

\subsubsection{Aportes del programa}

En cuanto a los aportes, las mujeres ponen en valor aspectos relacionados con el espacio de seguridad que implica el acceso a un dispositivo de vivienda, la importancia de la relación y el vínculo que se construye entre el equipo educativo y las mujeres, así como el desarrollo de redes que se generan entre las propias mujeres.

\section{Cobertura de necesidades básicas}

Cuando las mujeres solicitan el ingreso en los recursos residenciales de Lagun Artean, presentan situaciones de sinhogarismo encubierto donde tanto ellas como sus hijos/as a cargo carecen de medios para cubrir sus necesidades básicas. Es decir, la intervención se inicia en momentos de altos niveles de vulnerabilidad. Por ello, las mujeres ponen en valor la importancia de tener cubiertas las necesidades básicas de alimentación, techo, higiene, descanso, etc., tanto para ellas como para sus hijas/os:

Mis necesidades eran todo lo que ellos me ofrecieron. Necesidades básicas, tener un hogar, una casa, un techo donde dormir, alimento para poder darle a mi hijo. (Participante 4, Bolivia)

Cuando llegué a Lagun Artean, sinceramente te sientes persona, porque me pude dar una ducha, me pude bañar en condiciones... dormir tranquilamente. Comí... hasta me parecía tan irreal. Fue lo único que pensé, esto es mentira, cómo van a darme comida, que esté durmiendo en una cama, y a cambio de nada. Siempre pensaba que todo era mentira. (Participante 12, Nicaragua).

Espacio de apoyo emocional y seguridad Al ser preguntadas por lo que más valoran, la mayoría de las mujeres repite y destaca la palabra "seguridad". Es decir, en concordancia con varias autoras (Matulič et al., 2019), los programas residenciales actúan como elemento protector en sus procesos de exclusión residencial:

Lo más importante para mí era sentirme segura, que en Lagun Artean no podía pasarme nada. (Participante 5, Mali)

Estar recogida, con cariño, sin miedo, ya sabes miedo de fuera... nadie se va a aprovechar [...]. Por lo menos aquí estaba con gente de confianza... Necesitaba no sentir miedo. (Participante 9, Marruecos).

Además de poder tener cubiertas necesidades básicas, las mujeres destacan la importancia de ser recogidas y escuchadas; es decir, dan mucha importancia al apoyo emocional que reciben por parte del equipo educativo:

Aunque lo más importante sea dormir y comer a veces necesitas otras cosas como hablar, que te escuchen, sentirte acompañada... también son necesidades básicas. (Participante 1, Nicaragua)

Me sentía arropada con tanta gente, y además te consuelan y cuando estoy con ellos me siento menos triste, con menos problemas. (Participante 15 , Senegal)

En esa misma línea, las mujeres describen al equipo de educadores y educadoras como una familia y un referente en el que apoyarse. A pesar de vivir en un piso con normas, las mujeres destacan el clima familiar que se crea y cómo la relación entre las educadoras y ellas es muy cercana:

Yo la veo como alguien de mi familia, una amiga, no como una educadora. Eso es otro valor más. Necesitamos más que lo material, comer puedes comer en cualquier lado, pero esto... tienes una referencia. (Participante 8, Marruecos)

Sobre todo, el trato con la gente que entra, que no es un trato de yo soy educadora y tú eres chavala, y aquí las órdenes las doy yo. (Participante 1, Nicaragua)

\section{Apoyo mutuo entre las mujeres}

Además del apoyo por parte del equipo educativo, las mujeres destacan la red afectiva y el cuidado mutuo entre las propias compañeras en el interior del recurso residencial. Subrayan la función de apoyo que se dan mutuamente, incluso aunque no hablen el mismo idioma:

Aquí nunca me he sentido sola, estaban las demás compañeras; aunque no nos entendíamos por el idioma, no estábamos solas. (Participante 7, Argelia)

Me han recibido con los brazos abiertos, me sentía aliviada, como estar en una familia. Me quedaba con mis compañeras y no estaba sola. 
Hablábamos mal castellano, solo una hablaba castellano y francés hablábamos más o menos, pero disfrutábamos; parecíamos como hermanas. (Participante 15, Senegal)

Amistad y apoyo mutuo que en ocasiones continúa más allá del periodo de estancia en el recurso:

Y también yo he salido con una hermana, desde Lagun hasta hoy estamos juntas, todas mis cosas las sabe, ella es mi apoyo, me apoya en todo. Cuando tengo un problema, a la primera persona que llamo es a ella. (Participante 5, Mali)

Diversas autoras (Alter, 2008, Perondi, 2012, Damonti, 2014, y Sastre, 2015), señalan la falta de redes de apoyo como una de las características principales de las mujeres monomarentales en situación o riesgo de pobreza. En ese sentido, es fundamental promover desde los programas y recursos residenciales dinámicas que permitan crear redes de apoyo mutuo entre las propias mujeres.

\section{Acceso a formación y conocimiento de sus derechos} Otro de los aportes que destacan las mujeres es la posibilidad de acceso a cursos de formación durante su estancia en el recurso residencial. En el caso de las mujeres provenientes de África, resaltan la importancia de los cursos de aprendizaje del castellano. En general, las mujeres acceden a formaciones para la empleabilidad en sectores feminizados como hostelería, cuidados, puericultura, etc.; nichos laborales que son la principal, y mayoritariamente única, puerta de entrada al mercado laboral para las mujeres migradas (Oso y Parellas, 2012). Sin embargo, estos trabajos destacan por su precariedad, inestabilidad y poco reconocimiento social, lo que no garantiza la inclusión social de las mujeres.

Por otro lado, al mismo nivel que la formación, las mujeres entrevistadas valoran de manera muy positiva el conocimiento de sus derechos y el acceso a información sobre ellos que han tenido durante su estancia en el recurso:

Me ha hecho consciente de mis derechos; antes de llegar a Lagun no sabía que los tenía. (Participante 15, Senegal)

Ahora me siento más fuerte porque conozco qué es mío, cuáles son mis derechos. (Participante 13 , Sáhara)

Más allá de valorar la importancia que tiene para mujeres inmigrantes provenientes de otras culturas estar informadas sobre los derechos que tienen en las sociedades a las que llegan, nos parece fundamental que los programas de atención a mujeres inmigrantes y los agentes profesionales que intervienen no reproduzcan la construcción social extendida sobre la "mujer migrada" (Gregorio Gil, 2012) a partir de sus “carencias”, las cuales deben superar a través de formaciones (Galaz y Montenegro, 2015). Para estas autoras, esta construcción desde la carencia conlleva el riesgo de "asignar pasividad a quienes se encuentran en una posición de subalternidad y aprovechar esa subalternidad para colocarnos en una posición de superioridad y de relación (ma)paternalista" (Galaz y Montenegro, 2015: 1.673).

Posibilidad de ahorro y acceso a ayudas sociales Uno de los objetivos del programa es que, durante el tiempo de estancia en el recurso, además de formación las mujeres puedan ir teniendo acceso al mercado laboral y/o a la prestación de ayudas. En la mayoría de los casos, al trabajar en sectores feminizados muy precarizados tienen que completar su salario con prestaciones sociales o directamente al no encontrar trabajo acceder a la renta de garantía de ingresos (RGI). Debido a los requisitos cada vez más exigentes para poder acceder a estas ayudas, en el caso de la RGI, tres años de empadronamiento en el mismo lugar, estar en un recurso residencial les permite ir sorteando las dificultades para poder acceder a estas ayudas, además de poder ir ahorrando dinero para la salida. El acceso a la RGI es en muchas ocasiones la vía para poder salir del recurso:

Gracias a la RGI, me pude alquilar una habitación. (Participante 14, Guinea Ecuatorial)

En el caso de las mujeres extranjeras, hay que tener en cuenta la situación de desprotección que su estatus jurídico conlleva, lo que las expone a una situación de precariedad, inseguridad e indefensión respecto a los poderes públicos (Bedoya, 2000).

\subsubsection{Limitaciones del programa y de la red asistencial}

En lo que respecta a las limitaciones, las mujeres entrevistadas identifican, por un lado, limitaciones en cuanto al Programa Mujer de Lagun Artean y, por otro lado, señalan barreras encontradas dentro de la red asistencial y los servicios sociales.

\section{Limitaciones del programa}

Tres son las principales limitaciones que las mujeres apuntan: (1) una alta demanda permanente, (2) la falta de apoyo psicológico y (3) escaso seguimiento después de la salida del recurso. En cuanto a la duración del programa, varias de las mujeres entienden que el recurso de alojamiento es temporal y que debido a la alta demanda de mujeres solicitantes no pueden alargar tanto como quisieran su estancia. No obstante, sí que sienten que hubieran necesitado más tiempo, sobre todo para conseguir una mayor estabilidad emocional:

No me pareció tiempo suficiente, hubiera estado más tiempo porque no era tiempo todavía... porque las emociones... hay cosas que no... más complicadas cuando tienes hijos [...]. Ellos me 
dijeron de salir porque entraba más gente, para darle ayuda a otras mujeres... en su momento me pareció bien, pero... una mujer con un niño de un año... (Participante 12, Nicaragua)

En algunas vivencias de las mujeres se ha podido observar cómo el impacto del abandono del recurso supuso una revictimización en sus recorridos particulares. Las mujeres verbalizan la importancia de trabajar las necesidades emocionales y psicológicas con las que llegan. De hecho, las colocan al mismo nivel de importancia que las necesidades básicas de alojamiento y manutención:

Yo creo que estar bien no es solo tener trabajo o una habitación, es haber podido superar cosas que te han pasado. (Participante 4, Bolivia)

Cuando llegué necesitaba apoyo psicológico, no solo comida y una casa. (Participante 1 , Nicaragua)

En concordancia con otros autores (Maya, Martínez y García, 1999: 222), las dificultades y problemáticas que condicionan la experiencia de las mujeres migrantes no son solo a nivel legal, económico o cultural, sino que también manifiestan dificultades a nivel psicológico (estrés adaptativo, procesos psicopatológicos, duelos emocionales, habilidades sociales, etc.), que hacen mella en sus experiencias migratorias y que son, por tanto, problemáticas también de primer orden a trabajar y acompañar desde los programas de intervención social.

Por último, las mujeres subrayan la importancia de poder hacer un seguimiento más continuo después de su salida del recurso. Si bien es cierto que todas las mujeres entrevistadas afirman mantener un vínculo positivo con el equipo educativo de Lagun Artean, debido a la gran demanda que presenta la asociación, las mujeres carecen de un seguimiento sistemático cuando salen de los recursos. Es decir, las intervenciones están diseñadas de manera lineal, con un inicio y un final, sin contar con un trabajo de seguimiento y un trabajo de seguimiento organizado y continuo una vez las mujeres han dejado el programa:

Me hubiera gustado que al salir nos hubiéramos visto un poco más... de la noche a la mañana te quedas sola. (Participante 15, Senegal)

\section{Limitaciones de la red asistencial}

Atendiendo a las limitaciones encontradas no solo en la intervención de Lagun Artean, sino en el funcionamiento global de la red asistencial y los servicios sociales, se encuentran principalmente dos: (1) la persistencia de una mirada asistencialista y cortoplacista de la inclusión social y (2) una visión androcéntrica en el diseño y funcionamiento de albergues y recursos residenciales para personas sin hogar.

En primer lugar, la saturación de los servicios sociales tiene una correlación directa con el predominio de una atención asistencialista frente a modelos de intervención e inclusión más holísticos, que contemplan otras dimensiones como la psicológica, la relacional, la comunitaria o la participación social, entre otras.

Así, muchas de las mujeres salen de los recursos habiendo obtenido un empleo precario en el ámbito de los cuidados y las tareas del hogar y/o con alguna prestación social económica, lo cual no les garantiza una estabilidad personal, social y laboral. Como ellas mismas manifiestan, salen sin haber podido trabajar otros aspectos fundamentales para la inclusión social como el duelo migratorio, las relaciones de género, aspectos emocionales y personales que han quedado dañados (autoestima, seguridad personal, autopercepción corporal, etc.):

Yo no hubiera salido, no me veía preparada para irme sola, pero tenía dinero y tenía que dejar sitio a las que están peor. (Participante 9, Marruecos)

Salir fue muy duro, todavía tenía cosas dentro que me dolían. (Participante 12, Nicaragua)

En ese sentido, poner el foco de la intervención exclusivamente en lo laboral tiene como consecuencia una mirada reduccionista de los procesos de inclusión, corriendo el peligro además de intensificar la catalogación de "mujer inmigrada vulnerable" (Galaz y Guarderas, 2017), ya que los nichos laborales a los que pueden optar las mujeres son nichos precarios e inestables que no les garantizan una efectiva inclusión social, sino que las colocan en una cuerda floja de subsistencia y supervivencia diaria. Por lo tanto, cuando hablamos de inclusión social, no puede ser entendida exclusivamente como inclusión laboral.

Además, tener una mirada de la inclusión más allá del ámbito económico implica abandonar intervenciones homogéneas y normativas para todas las mujeres y apostar por una mirada más individualizada, que atienda las especificidades de las diversas situaciones por las que transitan las mujeres.

En segundo lugar, las mujeres identifican los recursos nocturnos de alojamiento como lugares hostiles e inadecuados, y mucho más para mujeres con hijos e hijas a cargo, debido a la gran cantidad de hombres que suelen ocuparlos y a la escasa intimidad que sienten:

Cuando llegué al albergue solo estábamos una mujer mayor y yo. Pero ella se fue y me quede sola, era la única chica. Me dio miedo dormir ahí la primera noche sola. (Participante 10, Camerún)

En concordancia con los testimonios de las mujeres, varios estudios señalan que los principales motivos por los que las mujeres no utilizan los servicios para personas sin hogar son: la presencia mayoritaria de hombres, la escasa adaptación a las necesidades específicas de las mujeres y el temor de la 
victimización (Emakunde, 2016), así como el estigma social que vincula el sinhogarimo con la prostitución (Pleace et al., 2016; Sales, 2016).

La falta de adaptación a las necesidades concretas de las mujeres se manifiesta, por ejemplo, en la falta de plazas para madres con niños y niñas a su cargo:

En el albergue me dijeron que no podían coger mujer con hijos, que no podía dormir ahí. (Participante 5, Mali)

En ese sentido, las mujeres identifican necesidades específicas por ser madres a la hora de diseñar recursos que les garanticen una seguridad a ellas y a sus criaturas:

Las necesidades pueden ser diferentes en el tema de si eres madre. No sabes cómo hacer frente a los miedos que tienes como madre, a no saber cómo hacerlo... (Participante 4, Bolivia)

Por tanto, se torna urgente potenciar la creación de recursos específicos para mujeres con hijos e hijas que, además de asegurarles protección, les ofrezcan un acompañamiento individualizado y una intervención integral.

\subsection{Situación actual}

Como señalábamos al inicio del artículo, uno de los objetivos que teníamos era poder tener una fotografía de la trayectoria de las mujeres antes, durante y después de pasar por el Programa Mujer de Lagun Artean, centrándonos en dos áreas claves: su situación económico-laboral y el acceso a una vivienda digna, dos áreas que condicionan la vida de las mujeres y las mantiene en la cuerda floja de la exclusión.

\subsubsection{Precariedad e inestabilidad económica continua: trabajos intermitentes y "suelo pegajoso"}

El Gráfico 2 muestra la actual situación sociolaboral de las mujeres.
Como se puede observar, el sector laboral del hogar y los cuidados es el que predomina entre las mujeres entrevistadas, teniendo que complementar varios trabajos con el cobro de la renta de garantía de ingresos:

Y me siento como una esclava. No tengo tiempo para pensar en mí, no me dejan tiempo. Trabajo en dos casas y cobro la parte complementaria que me corresponde de la RGI. (Participante 12, Nicaragua)

Por otro lado, tres de las mujeres entrevistadas están realizando estudios de carácter no reglado también dentro del ámbito de los cuidados y dos de ellas están percibiendo la RGI como única fuente de ingresos.

Por último, dos de las mujeres entrevistadas perciben la RGI sin estar sujetas a ninguna actividad formativa o laboral. Sin embargo, ambas afirman su intención de encontrar un trabajo que les permita desarrollar una independencia económica suficiente como para dejar de cobrarla:

Yo normalmente no pido la RGI, quiero hacer algo en la vida y ganar algo. (Participante 8 , Sáhara)

No me gusta cobrar RGI sin hacer nada, sin levantarme de la cama. Quiero trabajar. (Participante 11, Guinea Bissau)

Esto nos lleva a dos reflexiones importantes. En primer lugar, el actual mercado laboral se caracteriza por una marcada estratificación en función del género, el origen y el estatus migratorio en los países receptores de migración. En el caso del sector de los cuidados, además del género, las dimensiones de clase social, etnicidad o grupo nacional se entrecruzan, abocando a las mujeres inmigrantes a una participación laboral mayoritariamente precaria e inestable, que las coloca en los márgenes de la exclusión.

Precisamente en las entrevistas las mujeres resaltan la discontinuidad de sus empleos y la cantidad de estos, pero siempre ligados al sector de los cuidados o a la hostelería; trabajos de pocas horas

Gráfico 2. Situación sociolaboral actual de las mujeres entrevistadas

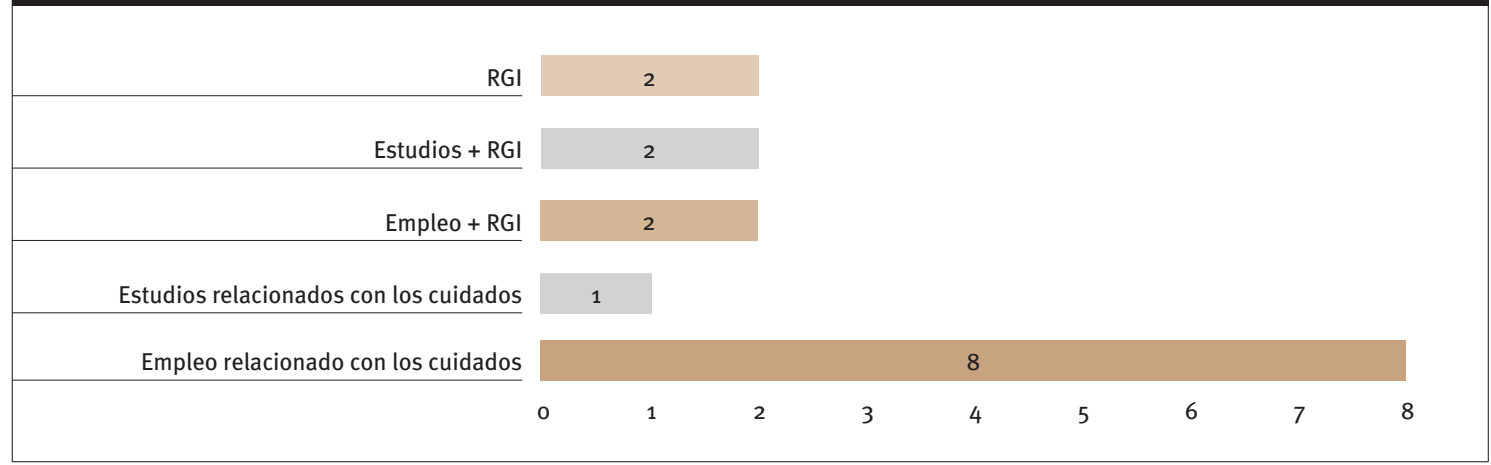

Fuente: Elaboración propia. 
intermitentes con pocas garantías de seguridad ni condiciones adecuadas para obtener residencias permanentes:

Y ahora pues aquí estoy, trabajo y no trabajo, a veces me llaman para ir a trabajar, a veces no me llaman, y así... (Participante 12, Nicaragua)

Tengo trabajo de dos horas haciendo de todo, trabajo cuando me llaman, aprovecho el tiempo al máximo cuando los niños están en el colegio. El trabajo es en una casa, en un domicilio. Estuve trabajando en un hotel, en un restaurante y también en turnos de noche cuidando personas mayores. (Participante 13, Sáhara)

Una segunda reflexión, por tanto, está relacionada con que, a medida que avanzan los años en la sociedad de destino, las mujeres son conscientes de las dificultades para desligarse del circuito del trabajo doméstico y de cuidados. Se crea así un círculo vicioso que dificulta su diversificación y movilidad laboral y las estanca laboral y económicamente. Este fenómeno, denominado "suelo pegajoso", implica que se vean adheridas a empleos vinculados a la reproducción social devaluados socialmente, lo que las encierra en un enclave laboral migrante caracterizado por salarios reducidos, empeoramiento de las condiciones laborales y dificultades de ascenso sociolaboral (Thayer, 2011).

\subsubsection{Dificultades de acceso a una vivienda digna y segura}

El acceso a una vivienda digna y estable es una dificultad central que atraviesa todas las narrativas de las mujeres, convirtiéndose en un gran estresor cotidiano que les impide una estabilidad emocional y personal. Las mujeres dan cuenta de su paso por diversidad de viviendas y, especialmente, las mujeres con hijos e hijas pequeñas tienen dificultades añadidas para encontrar una vivienda más estable en el tiempo:

A la gente no le gusta que vivas con niños pequeños... entonces te tienen un mes y tienes que buscarte otro sitio, estás otro mes en otro sitio, otro mes... depende de donde llegues te aguantan 3-4 meses... los niños son niños, se enferman, lloran... entonces es un poco complicado. (Participante 12, Nicaragua)

La gente no quiere vivir con niños, es lo primero que te preguntan cuando llamas. (Participante 1, Nicaragua)

Otra de las dificultades más nombradas es la posibilidad de tener acceso al padrón en los lugares de alquiler. El padrón, vía de acceso a derechos fundamentales como salud, educación, ayudas sociales, etc., sigue sin ser un derecho y muchas personas no permiten el empadronamiento en sus casas o suben el precio de los alquileres de las habitaciones para poderse empadronar:
Me fui al Casco Viejo, allí estuve también como un año viviendo con una chica que conocí en un curso que me mandó Lagun Artean de geriatría. Lo único que no me empadronaron. El dueño de la casa no empadronaba a nadie, y saliendo de Lagun Artean tienes que buscar sí o sí dónde empadronarte. (Participante 12, Nicaragua)

Asimismo, las mujeres dan cuenta de las dificultades que tienen para poder pagar los gastos de mantenimiento de la vivienda (luz, agua) y cómo tienen que subalquilar habitaciones del piso para poder hacer frente a los gastos:

En mi casa, ahora tengo un piso... no me gusta, pero estoy allí por mi niña, para estar con mi niña tengo que demostrar que tengo un sitio. Tengo gente viviendo en mi casa, dos chicos y tengo un subarriendo, pero con los gastos no llego, no puedo pagarlo todo porque te pagan el alquiler, pero comparado con los gastos... Siempre estoy retrasada. (Participante 12, Nicaragua)

En este sentido, las mujeres entrevistadas comparten el gran impacto personal que supone compartir piso con otras familias con las que no existe un vínculo afectivo y de confianza, y cómo en ocasiones se ven expuestas a situaciones de violencia. Es el caso de esta mujer entrevistada, que explica cómo un compañero de piso agredía a su hijo pequeño:

Ese chico le agarraba [a su hijo] de la mano y le metía sus uñas en las manos... le hacía mucho daño, él lloraba, él era pequeño [...] cuando me enteré no hice nada, no podía hacer nada. (Participante 12, Nicaragua)

Por otro lado, el rol de cuidadoras de las mujeres persiste dentro de la casa incluso conviviendo con personas con las que no tienen lazos afectivos ni familiares. Como sostiene Marcela Lagarde (2001), las mujeres arrastran el mandato de género de “ser para los/as demás” ignorando su propio autocuidado, lo que genera un gran impacto a nivel psicológico, emocional y relacional.

Por último, las malas condiciones de las viviendas, así como el hacinamiento, los abusos en los precios de las viviendas y el racismo imperante a la hora de conseguir un alquiler, hacen que tengan una gran movilidad de residencia, lo que no les permite poder desarrollar un proyecto personal y familiar seguro ni estable:

Tuve que cambiarme de piso varias veces porque las condiciones de la casa no eran buenas. (Participante 8, Sáhara).

Estaba en una habitación en la que casi no entraba la cuna de mi hijo, cuando ahorré un poco de dinero me cambié a otra. (Participante 13, Marruecos).

La casera no nos daba agua caliente y me cobraba quinientos euros. Cuando pude me fui. (Participante 12, Nicaragua). 


\subsection{Continuum de violencias}

Este círculo de precariedad y exclusión social en el que las mujeres están inmersas está atravesado por un continuum de violencias que muchas veces se inicia en sus países de origen, continua durante el tránsito a los países de destino y no cesa una vez que llegan, ya que la violencia institucional y racista la viven cotidianamente. Los cuerpos de las mujeres son claramente una herramienta de acceso y cruce de las fronteras durante el tránsito y la violencia sexual se convierte en muchos casos en moneda de cambio para poder cruzar las fronteras y continuar el viaje. En cuanto a la violencia institucional, la Ley de Extranjería condiciona enormemente la situación sociolaboral de las mujeres y las coloca en una situación de vulnerabilidad continua.

Desmenuzar las múltiples violencias que sufren las mujeres daría para otro artículo, pero sí que consideramos esencial visibilizar desde una mirada interseccional (Crenshaw, 1989) las violencias específicas que genera el cruce de diferentes ejes de opresión como el género, la raza, el origen, la clase, la situación administrativa irregular, etc., los cuales son expresión de estructuras sociales machistas, racistas y coloniales (Gandarias y Carranza, 2018). Esto implica que cualquier programa de intervención y acompañamiento tiene que abordar desde una mirada interseccional situaciones de vulnerabilidad y violencias ocultas. El hecho de tener recorridos vitales marcados por la violencia hace que las mujeres lleguen a naturalizarla, percibiéndola como algo prácticamente inevitable. Por eso, uno de los ejes principales de la intervención debe ser el acompañamiento en la reparación emocional, psicológica y comunitaria del continuum de violencias vividas.

\section{Conclusiones}

El objetivo de este articulo era analizar desde una mirada crítica los itinerarios de incorporación social de mujeres inmigrantes que han pasado por un programa específico de acompañamiento y un recurso residencial para mujeres con menores a cargo; centrándonos en dos áreas específicas: la laboral y el acceso a la vivienda. Desarrollamos a continuación las principales conclusiones.

En primer lugar, los quince itinerarios vitales analizados visibilizan la existencia de múltiples situaciones de exclusión social que no se enmarcan dentro de los parámetros convencionales de lo que se entiende por sinhogarismo. Como hemos visto en sus relatos, para no acabar durmiendo en la intemperie, a diferencia de sus homólogos los hombres, las mujeres despliegan estrategias relacionales vinculadas al espacio privado que genera un sinhogarismo oculto (más vinculado a lo que la tipología ETHOS considera vivienda insegura y vivienda inadecuada), más difícil de detectar y por lo tanto de atender. En el caso de las mujeres inmigrantes, estas estrategias relacionales se tornan más difíciles debido a su situación administrativa irregular, a las escasas redes de vínculos que tienen y a los prejuicios raciales existentes en torno a ellas, situación que se agrava aún más en el caso de mujeres con menores a cargo.

En segundo lugar, los programas específicos para mujeres monomarentales les permiten cubrir las necesidades básicas para ellas y sus hijos/as; suponen un espacio de seguridad y apoyo mutuo importante y son el trampolín para iniciar un itinerario de incorporación sociolaboral a través de cursos de formación y aprendizaje del castellano, conocimiento de sus derechos y acceso a ayudas sociales. No obstante, debido a la saturación y alta demanda de la red asistencial, se trata de programas con una mirada cortoplacista, cuyo foco de intervención se dirige mayoritariamente al ámbito laboral, atendiendo en menor medida otros aspectos fundamentales como la asistencia psicológica, la reparación del duelo migratorio o las múltiples violencias muchas veces normalizadas que arrastran las mujeres.

En tercer lugar, las mujeres inmigrantes se ven atrapadas en un círculo de precariedad y exclusión del que les es muy difícil escapar. Los itinerarios de formación e incorporación sociolaboral refuerzan los nichos laborales precarios, inestables y poco reconocidos socialmente, vinculados al sector de los cuidados y el trabajo del hogar, opciones laborales que no les permiten obtener una estabilidad económica y que las enclaustra laboralmente, lo cual revela una triple discriminación, resultado de la articulación de la clase social,el género y el origen. De la misma manera, las mujeres presentan múltiples dificultades para acceder a una vivienda estable y segura y se ven abocadas a una gran movilidad habitacional, hacinamiento y a la obligatoriedad de compartir vivienda con personas desconocidas, así como situaciones de violencia y racismo para poder acceder a un alquiler.

En síntesis, se torna necesario desarrollar programas específicos para mujeres inmigrantes con hijos e hijas a cargo, que no refuerce ni las aboque a un círculo de precariedad y exclusión continuo. Para ello, necesitamos programas de largo alcance, con una mirada holística e interseccional de la inclusión social más allá del ámbito laboral que aborde aspectos personales, relacionales y psicológicos, así como un acompañamiento comunitario en la reparación de las múltiples violencias que las mujeres sufren, pero también enfrentan. En ese sentido, queremos acabar incidiendo en la importancia de no configurar un perfil homogéneo y unívoco en cuanto a las trayectorias de las mujeres inmigrantes y, a pesar de los obstáculos estructurales, enfatizar su capacidad de agencia y su papel activo a la hora de desarrollar estrategias de subsistencia para ellas y sus familias tanto aquí como en sus países de origen. 


\section{Referencias bibliográficas}

ACIN, A. (2016): “¿Cuál es la diferencia entre los sin hogar y los sin techo?”, El Nacional.Cat, 17/06/2020, disponible en: 〈https://www.elnacional. cat/es/barcelona/sin-hogar-sin-techodiferencia_123654_102.html>.

AGUSTÍN, L.M. (2005): “Migrants in the mistress's house: Other voices in the "trafficking' debate", Social Politics: International Studies in Gender, State and Society, vol. 12, n 1, p. 96-117, 〈https:/// doi.org/10.1093/sp/jxio03〉.

ANTHIAS, F. (1998): "Rethinking social divisions: some notes towards a theoretical framework", The Sociological Review, vol. 46, n 3, p. 505-535, 〈https://doi.org/10.1111/1467-954X.00129〉.

ARRELS FUNDACIÓ (2017): Mitos y prejuicios acerca de las personas sin hogar, <https://www. arrelsfundacio.org/es/prejuicios/>.

BAPTISTA, I.; MARLIER, E. (2019): Fighting Homelessnes Sand Housing Exclusion in Europe: A Study of National Policies, European Commission, Bruselas.

BEDOYA, M.H. (2000): “Mujer extranjera: una doble exclusión: Influencia de la Ley de Extranjería sobre las mujeres inmigrantes", Papers: revista de sociología, n 6o, p. 241-256, <http://dx.doi. org $/ 10.5565 / \mathrm{rev} /$ papers/v6ono.1041>.

BIZITEGI (2018): Estudio sobre la realidad de las mujeres en situación de exclusión residencial, 〈https://www.bizitegi.org/wp-content/ uploads/2019/07/Mujeres-situacion-exclusionresidencial_cas.pdf〉.

BRUGUÉ, Q.; GOMÀ, R.; SUBIRATS, J. (2018): “De la pobreza a la exclusión social. Nuevos retos para las políticas públicas", Revista Internacional de Sociología, vol. 6o, no 33, p. 7-45, 〈https://doi. org/10.3989/ris.2002.i33.728>.
CARRASCO, L. (2014): Mujeres sin hogar en España: un análisis sociográfico desde una perspectiva feminista, Madrid, Universidad Complutense de Madrid, <https://eprints.ucm.es/26317/1/ TFM_MUJERES\%20SIN\%20HOGAR_LAURA\%20 CARRASCO.pdf $>$.

DAMONTI, P. (2014): “Una mirada de género a la exclusión social", Fundación Foessa: VII Informe sobre exclusión social y desarrollo social en Andalucía y España, p. 1-36, 〈https://www.foessa2014.es/ informe/uploaded/descargas/VII_INFORME.pdf〉.

EDGAR, B. y DOHERTY, J. (2001): Women and homelessness in Europe: Pathways, services and experiences, Policy Pr.

ENDERS-DRAGÄSSER, U. (2001): "Women, exclusion and homelessness in Germany", Women and Homelessness in Europe-Pathways, Services and Experiences, p. 207-18.

FERNÁNDEZ-RASINES, P. y GÁMEZ-RAMOS, T. (2013): “La invisibilidad de las mujeres sin hogar en España", Revista de Psicología, vol. 22, n 2, p. 42-52, 〈https://doi.org/10.5354/07190581.2014.30852>.

FUNDACIÓN ATENEA (2018): Los procesos de inclusión social desde la perspectiva de género: Mujeres y exclusión residencial. Más allá del sinhogarismo, <http://fundacionatenea.org/ http://fundacionatenea.org/wp-content/ uploads/2019/06/Informe-Exclusi\%C3\%B3nResidencial-de-las-mujeres.pdf〉.

FOESSA. F. (2014): VII Informe sobre exclusión social y desarrollo social en España, Madrid, Cáritas/ Fundación Foessa, 〈https://www.foessa2014. es/informe/uploaded/descargas/VII_INFORME. pdf〉.

- (2019): VIII Informe Foessa sobre Exclusión y Desarrollo Social en España, Madrid, Cáritas/Fundación 
Foessa, <https://caritas-web.s3.amazonaws. com/main-files/uploads/sites/16/2019/05/ Informe-FOESSA-2019-completo.pdf〉.

GALAZ V.C. y GUARDERAS A.P. (2017): “La intervención psicosocial y la construcción de las 'mujeres víctimas'. Una aproximación desde las experiencias de Quito (Ecuador) y Santiago (Chile)", Revista de Estudios Sociales, $\mathrm{n}$ 59, p. 68-82, 〈https://dx.doi.org/10.7440/ res59.2017.06>.

GALAZ, C. y MONTENEGRO, M. (2015): “Gubernamentalidad y relaciones de inclusión/exclusión: los dispositivos de intervención social dirigidos a mujeres inmigradas en España", Universitas Psychologica, 14 (5), p. 1.667-1.679, 〈https:// doi.org/10.11144/Javeriana.up14-5.grie>.

GANDARIAS, I. y CARRANZA, C. (2018): “Fronteras, violencias y cuerpos de mujeres en resistencia. Tiempos de feminismos: debates para la acción" Viento sur: Por una izquierda alternativa, $\mathrm{n} 161, \mathrm{p}$. 89-94.

GREGORIO GIL, C. (2012): “Tensiones conceptuales en la relación entre género y migraciones. Reflexiones desde la etnografía y la crítica feminista", Papers, 97 (3), p. 569-590.

INSTITUTO NACIONAL DE ESTADÍSTICA (2012): Encuesta a las personas sin hogar. Características sociodemográficas, 〈https://www.ine.es/ dyngs/INEbase/es/operacion.htm?c=Estadisti ca_C\&cid=1254736176817\&menu=ultiDatos\&i $\mathrm{dp}=1254735976608$ >

ÍNIGGUEZ RUEDA, L. (1999): “Investigación y evaluación cualitativa: bases teóricas y conceptuales", Atención Primaria, 23, 8, 496-502.

LAGARDE, M. (2001): Claves feministas para la negociación en el amor, Puntos de encuentro.

LAGUN ARTEAN (2018): Memoria general de datos, <http://www.lagun-artean.org/wp-content/ uploads/2019/05/Memoria-general-2018Versi\%C3\%B3n-cast.pdf〉.

MAYA JARIEGO, I.; MARTÍNEZ GARCÍA, M.F. y GARCÍA RAMÍREZ, M. (1999): “Cadenas migratorias y redes de apoyo social de las mujeres peruanas en Sevilla", Demófilo, 29, p. 87-106.

MATULIČ, M.V.; PASCUAL, A.M. y ZUERAS, I. (2020): "Sinhogarismo femenino: una aproximación a la intersección entre género, edad y procesos migratorios", Research on Ageing and Social Policy, vol. 8, n 1, p. 57-85, 〈http://dx.doi. org/10.447/rasp.2020.4724/.

MATULIČ, M.V.; DE VICENTE, I.; BOIXADÓS, A. y CAIS, J. (2019): "Las mujeres sin hogar: realidades ocultas de la exclusión residencial", Trabajo social global-Global Social Work: Revista de investigaciones en intervención social, vol. 9, n. 16, p. 49-68, 〈https://dx.doi.org/10.30827/ tsg-gsw.vgi16.8198>.

MARTINS, A. (2010): "Gender and homelessness: Homeless women in Lisbon", Homeless in Europe, vol. 6, p. 6-9.
MAYOCK, P. y SHERIDAN, S. (2012): “Women's 'Journeys' to Homelessness: Key Findings from a Biographical Study of Homeless Women in Ireland", Women and Homelessness in Ireland, Research Paper 1, School of Social Work and Social Policy and Children's Research Centre, Trinity College Dublin.

MINISTERIO DE SANIDAD, CONSUMO Y BIENESTAR SOCIAL (2015-2020): Estrategia Nacional Integral para Personas sin Hogar, Madrid, <https://www.mscbs.gob.es/ssi/ familiasInfancia/ServiciosSociales/docs/ EstrategiaPSH20152020.pdf〉.

OLABUÉNAGA, J.I.R. (2012): Metodología de la investigación cualitativa, Universidad de Deusto.

OSO, L. (2003): Estrategias migratorias de las mujeres ecuatorianas y colombianas en situación irregular: servicio doméstico y prostitución en Galicia, Madrid y Pamplona, Universidad Pontificia de Comillas, Madrid, p. 5-7.

- (2005): “Mulleres inmigrantes e prostitución en Galicia”, Cadernos de psicoloxía, vol. 1, n 1, p. 24-35.

OSO, L. y PARELLA, S. (2012): “Inmigración género y Mercado de trabajo: una panorámica de la investigación sobre la inserción Laboral de las mujeres inmigrantes en España", Cuadernos de relaciones laborales, vol. 30, n 1, p. 11.

PARELLA, S. y SAMPER, S. (2008): “Las estrategias de conciliación del ámbito laboral y familiar de las mujeres de origen inmigrante", La inmigración en la sociedad española: Una radiografía multidisciplinar, Ediciones Bellaterrra, p. 587606.

PERONDI, A.C. (2012): Familias formadas por una sola persona adulta con hijo (s) y/o hija (s) a su cargo: diagnóstico y propuestas, Madrid, Centro de Estudios Económicos Tomillo (CEET).

PLEACE, N. (2016): “Exclusion by definition: The underrepresentation of women in European homelessness statistics", en Women's homelessness in Europe, Palgrave Macmillan, p. $105-126$.

SALES, A. (2016): "Sinhogarismo y exclusión social. De la asistencia a la prevención”, RTS: Revista de treball social, $\mathrm{n} 209$, p. 9-25.

SALES, A.; GUIJARRO, L. (2017): "Dones sense llar: la invisibilització de l'exclusió residencial femenina", Revista Barcelona Societat, vol. 21, p. 81-89, <https://dx.doi.org/10.30827/tsggsw.vgi16.8198>.

TAYLOR, S. J. y BOGDAN, R. (1992): “La observación participante y la entrevista a profundidad", Introducción a los métodos cualitativos de investigación, Barcelona, Paidós, p. 108-111.

THAYER, E. (2011): “Trabajo y género: la condición social de inmigrante como referente para la definición de la identidad", Mujeres inmigrantes en Chile ¿mano de obra o trabajadoras con derechos?, p. $75-108$. 
